\title{
High resolution linkage maps of the model organism Petunia reveal substantial synteny decay with the related genome of tomato
}

\author{
Eligio Bossolini, Ulrich Klahre, Anna Brandenburg, Didier Reinhardt, and \\ Cris Kuhlemeier
}

\begin{abstract}
Two linkage maps were constructed for the model plant Petunia. Mapping populations were obtained by crossing the wild species Petunia axillaris subsp. axillaris with Petunia inflata, and Petunia axillaris subsp. parodii with Petunia exserta. Both maps cover the seven chromosomes of Petunia, and span 970 centimorgans (cM) and $700 \mathrm{cM}$ of the genomes, respectively. In total, 207 markers were mapped. Of these, 28 are multilocus amplified fragment length polymorphism (AFLP) markers and 179 are gene-derived markers. For the first time we report on the development and mapping of 83 Petunia microsatellites. The two maps retain the same marker order, but display significant differences of recombination frequencies at orthologous mapping intervals. A complex pattern of genomic rearrangements was detected with the related genome of tomato (Solanum lycopersicum), indicating that synteny between Petunia and other Solanaceae crops has been considerably disrupted. The newly developed markers will facilitate the genetic characterization of mutants and ecological studies on genetic diversity and speciation within the genus Petunia. The maps will provide a powerful tool to link genetic and genomic information and will be useful to support sequence assembly of the Petunia genome.
\end{abstract}

Key words: Petunia, linkage map, molecular markers, microsatellite markers, Solanaceae synteny.

\section{Introduction}

Petunia is an important horticulture crop cultivated for its flowers. It belongs to the Solanaceae family, which includes other important vegetable crops such as tomato, potato, pepper, and eggplant, as well as tobacco. Petunia is also a model plant for genetics and molecular biology. It has simple growth requirements, a short generation time, large genetic variability, and hundreds of seeds can be generated from a single cross. An efficient endogenous Ac/Ds-type transposon system has been successfully used to induce mutants and isolate the corresponding genes (Gerats et al. 1990; Stuurman and Kuhlemeier 2005). An easily screenable collection of transposon insertions has been created for reverse genetics (Vandenbussche et al. 2008). Petunia is easy to transform by stable Agrobacterium-mediated transformation (Conner et al. 2009), and an efficient virus-induced gene silencing system 
has been successfully established for functional analysis (Reid et al. 2009). Bacterial artificial chromosome (BAC) libraries are available for Petunia axillaris (Bossolini and Kuhlemeier, unpublished) and Petunia inflata (McCubbin et al. 2000; Puerta et al. 2009). Genetic maps of Petunia have previously been constructed using phenotypic markers (Gerats et al. 1993; Strommer et al. 2009), restriction fragment length polymorphism (RFLP) markers (Strommer et al. 2000), and amplified fragment length polymorphism (AFLP) markers (Strommer et al. 2002; Stuurman et al. 2004). The relatively large genome size of Petunia (1200-1500 Mbp, Mishiba et al. 2000) and low marker density has made map-based gene isolation efforts difficult, and only one example of positional cloning has so far been described (Bentolila et al. 2002). A genome sequence initiative has recently been undertaken (F. Quattrocchio and T. Gerats, personal communication 2010). The rich molecular tool box, together with a high genetic diversity and interesting biology (Gerats and Strommer 2009), makes Petunia an attractive model system.

Until now genetic mapping in Petunia was restricted to the popular Petunia hybrida. The designation $P$. hybrida refers to a large collection of garden varieties thought to be derived from interspecific crosses between Petunia integrifolia and $P$. axillaris. Petunia interspecific hybrids in nature are rare. Only hybridization between Petunia exserta and P. axillaris has been observed to some extent (Lorenz-Lemke et al. 2006). Genetic and cytological maps of several $P$. hybrida accessions have previously shown that their genome is differentiated by a number of genomic rearrangements (reviewed by Strommer et al. 2009). It is not known whether these rearrangements arose before or after domestication.

The most detailed gene-based map of Petunia dates to 10 years ago (Strommer et al. 2000), it spans 368 centimorgans (cM), and consists of 36 RFLP markers. The importance of Petunia as a model system and as a horticultural commodity makes it imperative to increase the genetic coverage of its genome. The aim of this work was to extend genetic mapping of the Petunia genome to wild species.

Our interest in wild Petunia species stems from the presence of closely related, cross-fertile species that display distinct pollination syndromes. Pollination syndromes are suites of floral characters, such as petal color, fragrance, reward production, and morphological traits that are adaptations to specific pollinators. The genus Petunia counts 14 species, all endemic to southern South America (Stehmann et al. 2009). Petunia axillaris and Petunia parodii are pollinated by nocturnal hawkmoths, $P$. inflata is pollinated by solitary bees, and $P$. exserta displays a typical hummingbird pollination syndrome (Fig. 1).

Mapping populations were obtained by the crosses $P$. axillaris $\times$ $P$. inflata and $P$. parodii $\times P$. exserta. We designed genederived codominant PCR markers that are highly reproducible, relatively inexpensive, and easily transferable across species. We describe for the first time the development and mapping of microsatellite markers in Petunia (Pm markers) derived from expressed sequence tags (EST) deposited in GenBank (www.ncbi.nlm.nih.gov/). Additional markers, including several genes encoding transcription factors and biosynthetic enzymes, were developed from sequences of genes with a putative role on floral development and color or scent biochemistry. Further marker saturation was achieved with multilocus AFLP (Vos et al. 1995) analysis or with markers designed to target specific Petunia chromosomal regions, relying on synteny with the related genome of tomato (Pt markers). We compared the mapping position of the markers of Petunia with that of tomato, and we provide a first insight into the extent of macrosynteny between these two representatives of the Solanaceae.

\section{Materials and methods}

\section{Plant material and DNA extraction}

Petunia axillaris subsp. axillaris (accession N), P. axillaris subsp. parodii (accession S7), and P. inflata (accession S6) were kindly provided by R. Koes, Department of Genetics at the Free University Amsterdam, Amsterdam, the Netherlands. Petunia exserta was a gift from R. Griesbach, Beltsville Agricultural Research Center, USDA, Beltsville, Maryland. The plants were grown in the greenhouse and manually crossed. Two mapping populations derived from interspecific crosses were developed for genetic analysis. A first mapping population consisting of $173 \mathrm{~F}_{2}$ plants was created by selfing a single $\mathrm{F}_{1}$ plant that was obtained from the cross $P$. exserta $\times$ $P$. parodii. A second mapping population of $176 \mathrm{BC}_{1}$ plants was derived from backcrossing a $\mathrm{F}_{1}$ plant $(P$. axillaris $\times P$. inflata) with $P$. axillaris as a seed parent. In this second population, the backcross design was chosen to avoid segregation distortion at the self-incompatibility $S$-locus of $P$. inflata. All seedlings were sown in germination pots and transplanted into single pots at the stage of $\sim 3 \mathrm{~cm}$. DNA was extracted following a cetyltrimethylammonium bromide (CTAB) extraction (Murray and Thompson 1980) when the plants were 3 weeks old.

\section{Marker design and genotyping}

Petunia EST sequences were downloaded from GenBank and mined for microsatellite motifs using the software SPUTNIK (Abajian 1994). To avoid amplification problems, repeats including cytosines and guanosines exclusively were not considered. We designed PCR primers preferentially targeting perfect repeats. Microsatellite markers were PCR amplified from petunia genomic DNA of the species $P$. axillaris, $P$. inflata, $P$. exserta, and $P$. parodii. Primers are listed in Table 1. A summary of the polymorphisms detected is provided in the Supplementary data ${ }^{1}$ (Fig. S1). To reduce genotyping costs, forward primers were labeled as described by Schuelke (2000). The amplification products were electrophoresis separated and visualized on a LI-COR 4300 DNA sequencer (LI-COR Biosciences, Bad Homburg, Germany). All markers were amplified with the same PCR conditions in $10 \mu \mathrm{L}$ volumes, containing approximately $20 \mathrm{ng}$ of template DNA, $1 \mu \mathrm{L}$ PCR buffer $(60 \mathrm{mmol} / \mathrm{L} \mathrm{KCl}, 12 \mathrm{mmol} / \mathrm{L}$ Tris$\mathrm{HCl}, \mathrm{pH}$ 9), $2 \mathrm{mmol} / \mathrm{L} \mathrm{MgCl}_{2}, 0.35 \mathrm{pmol}$ of the M13-tailed forward primer, 3.5 pmol reverse primer, 1.7 pmol labeled (IRD-700/800) M13 primer (5'-CACGACGTTGTAAAACGAC), $0.2 \mathrm{mmol} / \mathrm{L} \mathrm{dNTPs}$, and $0.5 \mathrm{U}$ of Taq polymerase. Thermocycling started with a denaturation step for $3 \mathrm{~min}$ at $96{ }^{\circ} \mathrm{C}$ followed by 45 cycles of $15 \mathrm{~s}$ at $96{ }^{\circ} \mathrm{C}, 30 \mathrm{~s}$ at $50{ }^{\circ} \mathrm{C}$,

${ }^{1}$ Supplementary data are available with the article 
Fig. 1. Petunia species produce flowers adapted to different pollinators. Petunia axillaris (both subsp. axillaris and parodii) are adapted to nocturnal hawkmoth, while Petunia inflata attracts diurnal bees. Petunia exserta exhibits red flowers typical for hummingbird visitation. Pictures from A. Dell'Olivo and M. Gremillon.

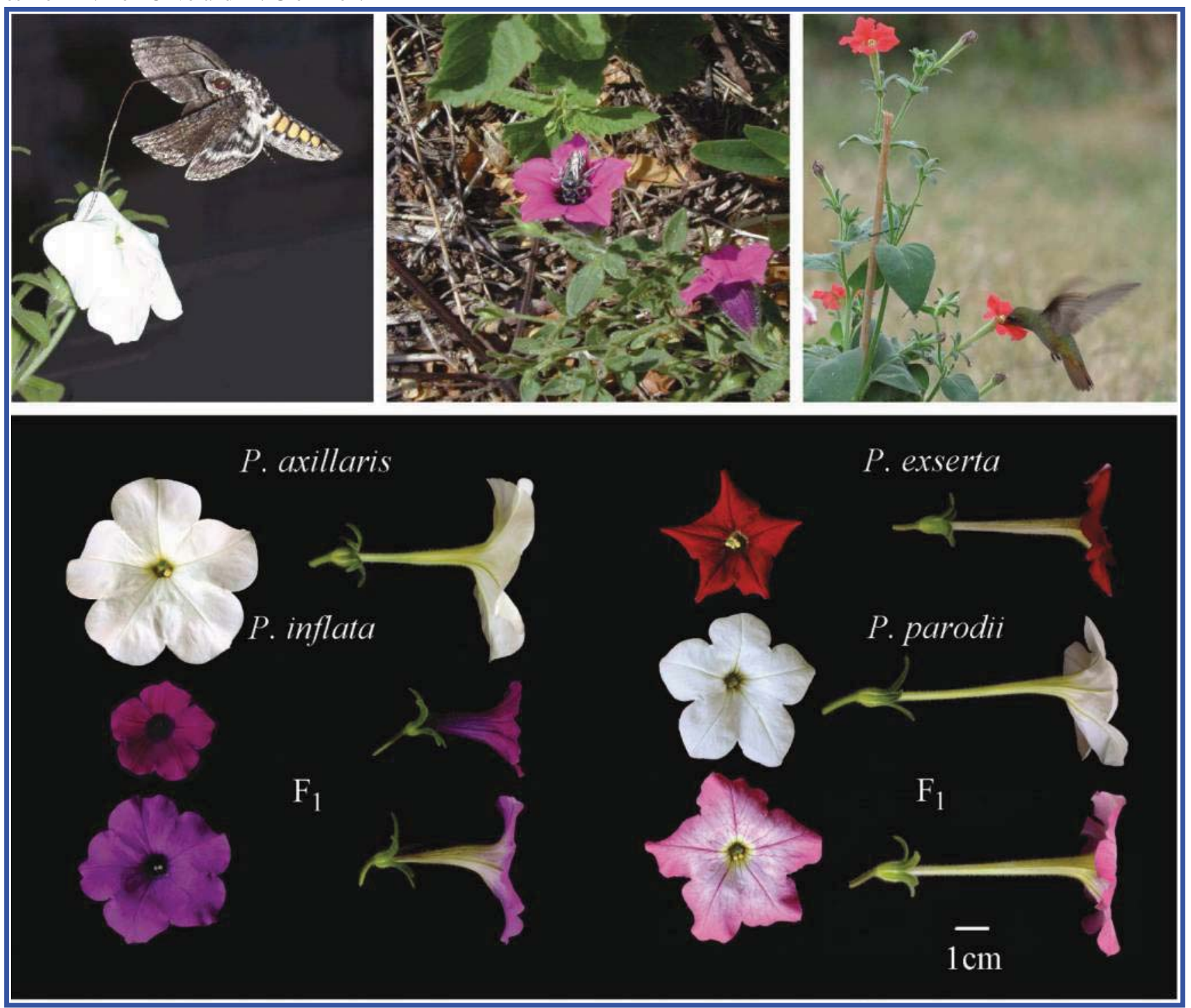

and $1 \mathrm{~min}$ at $72{ }^{\circ} \mathrm{C}$, and stopped after a final extension step of $72{ }^{\circ} \mathrm{C}$ for $7 \mathrm{~min}$. After PCR, samples were denatured by adding $30 \mu \mathrm{L}$ formamide stained with bromophenol blue. Chromosomal location of the polymorphic markers was determined by genotyping $173 \mathrm{~F}_{2}$ segregant lines obtained from a cross between $P$. exserta and $P$. parodii and $176 \mathrm{BC}_{1}$ lines obtained from a cross between $P$. axillaris and $P$. in flata. The polymorphic index content (PIC) for the multiallelic microsatellite markers was inferred from parental tests on the four wild species $P$. axillaris $\mathrm{N}, P$. parodii $\mathrm{S} 7, P$. exserta, and $P$. inflata S6. It was calculated as $\mathrm{PIC}=1-\sum_{i=1}^{n} p_{i}^{2}, p_{i}$ being the frequency of the allele $i$ at the marker locus $p$.

PCR primers used for genotyping the cleaved amplified polymorphic sequence (CAPS) markers were obtained from the literature or GenBank, and the sequences for the markers AN2, AN4, MYBB, and MYBX were kindly provided by F. Quattrocchio, Free University Amsterdam. Amplification was performed following a PCR protocol in $20 \mu \mathrm{L}$ volume containing
$50 \mathrm{ng}$ of template DNA, $2 \mu \mathrm{L}$ PCR buffer $(60 \mathrm{mmol} / \mathrm{L} \mathrm{KCl}$, $12 \mathrm{mmol} / \mathrm{L}$ Tris- $\mathrm{HCl}, \mathrm{pH} 9), 2 \mathrm{mmol} / \mathrm{L} \mathrm{MgCl}_{2}, 20 \mathrm{pmol}$ of forward and reverse primer, $0.2 \mathrm{mmol} / \mathrm{L}$ dNTPs, and $1 \mathrm{U}$ of Taq polymerase. PCR products were digested with the appropriate endonucleases as indicated in Table 2, adding a mix of $4 \mu \mathrm{L}$ water, $0.5 \mu \mathrm{L}$ of the appropriate buffer, and $0.5 \mu \mathrm{L}$ of the enzyme directly to $10 \mu \mathrm{L}$ of PCR reaction. Digested fragments were electrophoresed on agarose gels and stained with ethidium bromide. AFLP markers were genotyped using the same settings as described by Strommer et al. (2002). We tested six AFLP primer combinations designed with EcoRI-MseI and eight with PstIMseI. Generally, primers designed on Pst $\mathrm{I}-M s e \mathrm{I}$ resulted in fewer amplicons than EcoRI-MseI. To compensate, the amplification reaction was performed with only two selective nucleotides at the MseI primer. The AFLP markers were separated with a LI-COR sequencer. As the AFLP markers were dominant, they were added only to the $P$. axillaris $\times$ P. inflata $\mathrm{BC}_{1}$ cross. 


\begin{tabular}{|c|c|c|c|c|c|c|c|}
\hline Chr. & SSR & Forward primer & Reverse primer & EST & Putative function & PIC & SSR motif \\
\hline 1 & PM15 & GTGGCTGGCAACATTGACTA & CACTTACCCCTCAGTCCTCG & CV297655 & Unknown & 0.75 & $(\mathrm{CT})_{12}$ \\
\hline 1 & PM37 & GGGGTGGGAATTCTAGTGGA & TGGATGAGCCATAATCTTTGC & EB174496 & Transcription factor & 0.625 & $(\mathrm{ATG})_{6}-(\mathrm{CAA})_{5}$ \\
\hline 1 & PM41 & GGCTCAAACACAATTTCCTC & CTCCAACAAAGTTACTTGCAG & CV297469 & Jasmonate ZIM domain & 0.625 & $(\mathrm{TC})_{11}$ \\
\hline 1 & PM42 & CGGCTCAAACACAATTTCCT & AATTCAACCGCCATGAAGTC & CV297469 & Unknown & 0.625 & $(\mathrm{TC})_{11}$ \\
\hline 1 & PM81 & ACTGAAATCGTTGGGCGTT & AAAAGGAGTTGCATATCCTGATTA & CV292797 & Unknown & 0.625 & $(\mathrm{~T})_{16}$ \\
\hline 1 & PM83 & GCAAGTGTTCCATCTTGTC & CTCTGACCAAATAATGTG & CV299390 & Unknown & 0.625 & $(\mathrm{~T})_{19}$ \\
\hline 1 & PM85 & TGCAAATGAATGTCCAGGAT & TGCTGCAACTTTCCCAATTA & CV294317 & Unknown & 0.625 & $(\mathrm{TA})_{7}$ \\
\hline 1 & PM101 & GAGAGAGAACCCTAACCC & GCAGAAGAAACAGAGATCC & FN001660 & Unknown & 0.667 & $(\mathrm{CTT})_{6}$ \\
\hline 1 & PM120 & GGTTTAGATACTGAAGTTG & CCAGCATTACACCAACCTG & FN005966 & Unknown & 0.625 & $(\mathrm{~T})_{16}$ \\
\hline 1 & PM149 & ССТААТСАAАCACGTAАСТC & GGATGATGACACGTGGATCG & FN042637 & Tyrosine phosphatase & 0.625 & $(\mathrm{CT})_{10}$ \\
\hline 1 & PM164 & GGGGATGGCTACAGCAGC & CTTGCAGCTCATGGCAAAGC & FN014610 & F-box family & 0.375 & $(\mathrm{CAG})_{6}$ \\
\hline 1 & PM169 & GCAGAGAAACTACACTAATAGGG & CCTGAGGAAGAGCAGCAGC & FN016284 & Nucleic acid binding & 0.75 & $(\mathrm{CA})_{12}-(\mathrm{CAG})_{8}$ \\
\hline 1 & PM188 & CCCAACCATTGGCTACAGCC & GGACAACACAATACAATCTCTGC & FN037917 & Singlet oxygen response & 0.75 & $(\mathrm{CTT})_{8}$ \\
\hline & PM193 & CGCAACATCACCACTATCAG & GCTGCCAAGTCCGACAATGG & FN030612 & Unknown & 0.625 & $(\mathrm{CAT})_{6}$ \\
\hline & PM195 & GCCTTTCGCCGCTGTCACTG & GAGCAAATCGTGACCGTTGG & FN026706 & Phosphatidylserine synthase & 0.625 & $(\mathrm{GAA})_{6}$ \\
\hline & PM13 & GAAGGCAAGAATAGTCACC & CCGATTACTGTTTGAGGAGG & CV296369 & Unknown & 0.375 & $(\mathrm{CAC})_{9}$ \\
\hline & PM21 & CTACCGGTAGGCAGTAGTTGC & CCTCGACCTTCTTCCTGAC & CV297594 & KDR transcription regulator & 0.75 & $(\mathrm{TAC})_{8}$ \\
\hline & PM32 & TTCTCTAAGAAGAAACAATAAAGCTCA & GGCTATGCCAGCTTTGGTAA & CV298848 & Fascilin-like precursor & 0.625 & $(\mathrm{~A})_{23}$ \\
\hline & PM76 & GATCGCAACCTGGATCCTAA & AGGGCTGCACTCTTGTTTGT & CV297778 & Unknown & 0.375 & $(\mathrm{GAACCC})_{6}$ \\
\hline & PM88 & CTGTTTCCTAATTACCTG & GCCACTGGCATGGCTGCA & CV298718 & Unknown & 0.625 & $(\mathrm{TA})_{7}$ \\
\hline & PM93 & GCACCTCAGGCTGGTGCACC & GCAGTTGAAACAGAGGGACC & DC243258 & Unknown & 0.667 & $(\mathrm{TGC})_{6}$ \\
\hline & PM94 & CCGTGTTAGTATTGCCCAGG & CTCTAGATTGACCATAGC & CV300671 & Photoassimilate response & 0.625 & $(\mathrm{GGT})_{6}$ \\
\hline & PM111 & CACCATGAGGAACATCAAGC & GGAACTGGCTGAGGGAAACC & FN000621 & Unknown & 0.375 & $(\mathrm{CAC})_{9}$ \\
\hline & PM113 & GGCTCTGTCTGCAATGGACG & CTCTAGATTGACCATAGC & FN032542 & RNA binding & 0.375 & $(\mathrm{TGG})_{6}$ \\
\hline & PM119 & CCGACACATACCAATTCAC & CACCTAACGTACATTAGC & FN004737 & Inositol triphosphate kinase & 0.75 & $(\mathrm{TG})_{9}$ \\
\hline & PM124 & СССАССАССАСТТССАТТСАС & CGATGCTTGATTCCCCAC & FN005227 & Unknown & 0.375 & $(\mathrm{TGA})_{7}$ \\
\hline & PM142 & GGTGGTGCTGAGCCAAAGC & CGCCAGCTGCTTCTGAG & FN008269 & Transcription factor & 0.375 & $(\mathrm{ACT})_{6}$ \\
\hline & PM165 & СТСТАССТСТАСАТСТАСС & GTGCAGCGACAACGAGTC & FN045187 & UBX-domain & 0.375 & $(\mathrm{CAA})_{6}-(\mathrm{GAA})_{6}$ \\
\hline & PM186 & CCTTTACTAGTCTCAGAATTGC & GGATAATGATGATGACCC & FN036047 & TCP-like transcription factor & 0.375 & $(\mathrm{GTT})_{7}$ \\
\hline 2 & PM190 & CGAGTTGATGGTGCAATTGTG & CTAGAAAGTTCCTCCGG & FN038900 & TraB family & 0.625 & $(\mathrm{GAA})_{7}$ \\
\hline 2 & PM200 & CCTGACCCTCCCAGAACC & GGTAACATCTCCCTCACTTCC & FN022583 & Unknown & 0.667 & $(\mathrm{GTT})_{6}$ \\
\hline 2 & PM202 & СCCTGTTTCTTCTTCAC & CATCCACCACTTGTTGTTGAG & FN031565 & Transcription factor & 0.375 & $(\mathrm{ATT})_{7}$ \\
\hline 2 & PM205 & GGAGCCAAGTTGCTTGAGG & CССТTCAACAACACCACC & FN035754 & Transcription factor & 0.625 & $(\mathrm{TTG})_{6}$ \\
\hline 3 & PM12 & GGAACCTTCACAGAATGG & GAAGTTGTTGGCTCAACC & CV293902 & Unknown & 0.5 & $(\mathrm{CAA})_{7}$ \\
\hline 3 & PM79 & TTGGCGAAACATGCTGTTAG & CAAGCTTCATAAACCAACAACC & CV299471 & HTB4 DNA binding & 0.625 & $(\mathrm{GTT})_{5}-(\mathrm{A})_{11}$ \\
\hline 3 & PM99 & CAAGTGTTGGAGCAGTGGAA & GCCCTGCAAAAATGTGAAAT & CV294997 & Unknown & 0.375 & $(\mathrm{TTC})_{5}$ \\
\hline 3 & PM183 & CCTATTTCAGTCCATGAGGC & GTTAGCTGTCTGCTGATCAC & FN041860 & Phosphatase & 0.75 & $(\mathrm{GT})_{10}$ \\
\hline 3 & PM191 & GGAGAAGATTGTTGGTAAC & GGGAAACGATCTCTTGCTG & FN034502 & Unknown & 0.75 & $(\mathrm{TTG})_{6}$ \\
\hline 3 & PM197 & CCATAAGTGAAGGATCCTGC & CTGACAACTTACACAGGAACAC & FN032593 & Nucleic acid binding & 0.375 & $(\mathrm{CCA})_{7}$ \\
\hline 3 & PM219 & GCTGTAACATGTAGCTGTG & GGCTGCCAATCCATGCAGTC & FN014488 & Unknown & 0.445 & $(\mathrm{TGA})_{8}$ \\
\hline 4 & PM7 & CGTTTTTCATTGCATTGTCG & CGTTTCCCTCCTTTGATCTG & CV298460 & Squalene monooxygenase & 0.75 & $(\mathrm{AAC})_{10}$ \\
\hline 4 & PM8 & TCTGCAAACTTCAAAGCCAA & ACATGCCATGCACTTTTGAG & CV298210 & Transcription factor & 0.75 & $(\mathrm{AAGA})_{10}$ \\
\hline
\end{tabular}




\begin{tabular}{|c|c|c|c|c|c|c|c|}
\hline Chr. & SSR & Forward primer & Reverse primer & EST & Putative function & PIC & SSR motif \\
\hline 4 & PM40 & AGCTTCCTTTTTGAGCCACA & TGGCTTAAGCAAGACAATGG & CV299350 & Mannan synthase & 0.75 & $(\mathrm{~T})_{14}-(\mathrm{A})_{10}$ \\
\hline 4 & PM63 & TGGTACAATGGAGCAGAGG & ATGTGAGATTCCCAACGACC & NP1240021 & Transcription factor & 0.75 & $(\mathrm{ACAGCA})_{5}$ \\
\hline 4 & PM68 & GTCGAACGAGGATCATGTC & GTGTCCTCCTAATGCTTGCC & NP1240041 & Transcription factor & 0.375 & $(\mathrm{CAA})_{6}$ \\
\hline 4 & PM77 & ACCACGAGAAGAAGGAAGCA & CGAACAACGAGTTAAACCCC & CV298105 & Glycine-rich protein & 0.667 & $(\mathrm{GT})_{9}$ \\
\hline 4 & PM98 & ATGGAGGTAGCAAATGCAGG & CAACCAAATGCAGCTTCAGA & CV298392 & Methionine sulfoxide reductase & 0.5 & $(\mathrm{ATT})_{5}$ \\
\hline 4 & PM107 & GTCAAAGGTTGCAATCTCT & TGTTGCTGATGAGCAGTAG & FN001301 & Transcription factor & 0.625 & $(\mathrm{CAA})_{8}$ \\
\hline 4 & PM155 & GGCAACGACAATGGTGG & CCTTTGATCTGCATTCTCC & FN010739 & Squalene epoxidase & 0.667 & $(\mathrm{AAC})_{10}$ \\
\hline 4 & PM166 & GGCACTTGATTGTCCTTGTG & CCATGAATCGAATGCAG & FN014864 & Unknown & 0.625 & $(\mathrm{~A})_{32}$ \\
\hline 4 & PM173 & CAGCGCTATCAACAGCAG & GTGAGAGGCAAGTGATTGG & FN039910 & Unknown & 0.667 & $(\mathrm{GCA})_{6}$ \\
\hline 4 & PM181 & CCAGCTCTCTCGGAGCTG & CATCAACTATGTAGGAC & FN000364 & Harpin-induced protein & 0.375 & $(\mathrm{GAT})_{6}$ \\
\hline 4 & PM187 & GAGGCATTGTCACAGGC & CTATGGATGCAGCTAGGCCAC & FN031819 & Unknown & 0.5 & $(\mathrm{GCT})_{6}$ \\
\hline 4 & PM206 & CAGTGGCAGATGGAAGAGC & CCAACGCAAGCAAACAAGGC & FN035807 & Allantoate amidohydrolase & 0.75 & $(\mathrm{TC})_{12}$ \\
\hline 4 & PM218 & GTAGGACAAAAGTAAAGGGTG & GTTTGAAGTTAGACAATCG & FN018261 & NHL1 domain & 0.375 & $(\mathrm{CAT})_{6}$ \\
\hline & PM19 & ACCCTTGGAAAATGTCGTTG & TTCAAATTTCATCAGTGGCG & CV297851 & Unknown & 0.75 & $(\mathrm{~T})_{12} \mathrm{c}-(\mathrm{T})_{16}$ \\
\hline & PM44 & AGAATCCCCATATGCTCCG & AGCAGCACCAACAACACAAG & CV298575 & Pectinesterase & 0.625 & $(\mathrm{~A})_{15}-(\mathrm{A})_{10}$ \\
\hline & PM71 & CACTAGGACTCCTATTTCAC & GCTTATAAGGGAAGAGACTG & CV298122 & Unknown & 0.75 & $(\mathrm{CT})_{8}$ \\
\hline & PM72 & GTAAAGCCGTTTTGTTGGGA & CATTGAGGACTCTGCGATTG & CV295851 & Unknown & 0.375 & $(\mathrm{CT})_{9}$ \\
\hline & PM110 & GGTACAGGGCTAGCAGG & CTAGTTGGGTGTTCACAG & FN006325 & Disease resistance & 0.667 & $(\mathrm{AAC})_{8}$ \\
\hline & PM167 & CTCACTAACCAАСТTCACC & CTAAGAAGCTTAAGAGTG & FN015039 & Glycosyl transferase & 0.75 & $(\mathrm{TTC})_{12}$ \\
\hline & PM177 & СССТТАСТСТСТТСТТСАСС & GAACTATGAACCATAGCTCTC & FN016759 & Unknown & 0.667 & $(\mathrm{CA})_{11}$ \\
\hline & PM179 & CGGAGGAGGAGGACAAGGC & CCAGTTGCTCAGCCGATTGC & FN019539 & RCD1-like cell differentiation & 0.445 & $(\mathrm{AAT})_{6}$ \\
\hline & PM192 & GCTGCTTTAAGATTCAGAGGC & CTGAACTTTGCATTGGC & FN036401 & Transcription factor & 0.75 & $(\mathrm{CAG})_{8}$ \\
\hline & PM210 & CCTTGTGGCATAAGCTGCC & CCAACAACTGCAACAGCAGC & FN042698 & MYB transcription factor & 0.625 & $(\mathrm{TTG})_{6}$ \\
\hline & PM9 & GGAGGAGGAATATGAAGAAGC & СТСТTCTTCCTCCTCCGCAG & CV300118 & Unknown & 0.75 & $(\mathrm{AGA})_{17}$ \\
\hline & PM17 & TCCATCTCGTTTAGCAACCA & GGCTTCCAGCAAGAGAAGTG & CV301045 & Unknown & 0.625 & $(\mathrm{CTG})_{8}$ \\
\hline J & PM66 & CTATGGGAACTTCTCCTAAC & GCATTTCTAGGTTCTAGAGG & CV301241 & Unknown & 0.667 & $(\mathrm{AT})_{7}$ \\
\hline 6 & PM90 & TGGCGCTGAAACATTCTATG & AGAAGACAACGACAACGCAA & CV297287 & Acyltransferase & 0.445 & $(\mathrm{TC})_{7}$ \\
\hline 6 & PM91 & AGCCAGGCAAAGACCATTTA & TTTCACGTCATAATCCACGG & CV298703 & Transcription factor & 0.667 & $(\mathrm{TC})_{7}$ \\
\hline 6 & PM105 & CAGTAGGAAGGGTGCAGTGG & GTGCACGGAAGTTCTCG & FN001497 & Peptidyl-prolyl isomerase & 0.667 & $(\mathrm{~A})_{18}$ \\
\hline 6 & PM106 & GTTCCTCCAGGCACTTCTGG & CAGAGAGGACACAACTCCTC & FN004825 & Gibberellin-regulated protein & 0.75 & $(\mathrm{~T})_{17}$ \\
\hline 6 & PM117 & CCATACCCCATCTTCCACTGG & GGTGGCAACCTTGAGCTCC & FN004482 & Kinase & 0.445 & $(\mathrm{GTG})_{7}$ \\
\hline 6 & PM132 & GCAGTAGGGCATTGCAG & CTGATTCCTCCTCCAGCTCGAG & FN010866 & Cytochrome $b-f$ synthesis & 0.375 & $(\mathrm{ATC})_{6}$ \\
\hline 7 & PM33 & AАAАТTССТTTTTCTCTTTTCTTCC & GCAATACCGGTCCACTTGAT & CV298303 & Serine-rich protein & 0.625 & $(\mathrm{~A})_{24}$ \\
\hline 7 & PM54 & CCGAAACCCAGGAAACGC & CCTGTTTGATGTCAAACCC & CV299353 & Unknown & 0.667 & $(\mathrm{~A})_{18}$ \\
\hline 7 & PM103 & GTGGATGACAAACTTGAGG & GACAGCAGTGGTGTTTGG & FN006664 & Cystathionine beta-lyase & 0.75 & $(\mathrm{~A})_{20}$ \\
\hline 7 & PM144 & GCAGCCCTTCTTCACTG & CCATTGAATCCACAAGG & FN008495 & Unknown & 0.625 & $(\mathrm{~A})_{20}$ \\
\hline
\end{tabular}




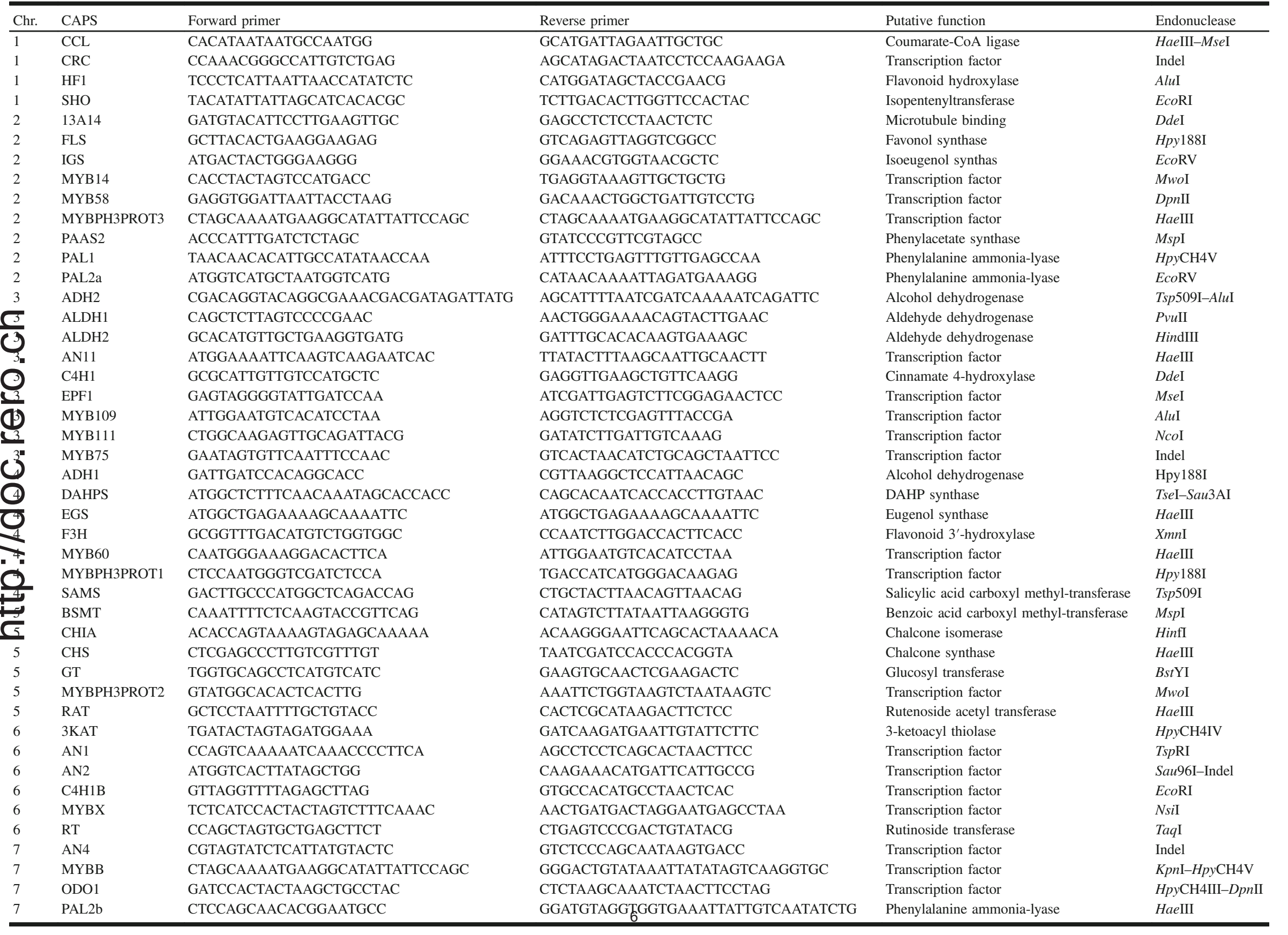


To further improve genetic mapping of some specific regions of the seven Petunia chromosomes, we relied on the genome of tomato (assembly 2.10, solgenomics.net, Müller et al. 2005). Sequences of the Petunia markers were located in the tomato genome with BLASTN. Site-specific Petunia markers (designated as Pt, Table 3) were designed from those regions that were poorly targeted in tomato. The Pt markers were genotyped with the same settings of the microsatellites when the source of polymorphism was a short insertiondeletion and with the same settings of the CAPS markers when a digestion was necessary. Gel pictures of the parental lines for the markers, DNA sequences, and additional genotyping details are available at www.botany.unibe.ch/deve/ caps/index.html. The putative function of all the gene-derived markers relied on Petunia literature information when available or alternatively was inferred from a BLASTX search in the Arabidopsis protein database (TAIR9, www. arabidopsis.org/).

\section{Linkage mapping}

Linkage was determined with MAPMAKER 3.0b (Lander et al. 1987). Recombination fractions were converted into Kosambi centimorgan units (Kosambi 1944). The best marker order within each linkage group was resolved with the ripple command. Uncertain marker orders were manually refined after correcting genotyping errors and later confirmed with the ripple command of the R-library R/qtl (R Development Core Team 2010; Broman and Sen 2009). Linkage groups were drawn with MapChart (Voorrips 2002). Chromosome definition and orientation for the linkage groups was inferred from previously published cytological and genetic maps (Gerats et al. 1993; Strommer et al. 2000; Strommer et al. 2009).

\section{Assessment of Petunia-tomato synteny}

The degree of synteny between tomato and Petunia was estimated independently for each of the two Petunia maps with the assembled tomato genome (Solanum lycopersicum assembly 2.10, solgenomics.net). The maps of Petunia and the tomato genome sequence were linked together with a BLASTN search (Altschul et al. 1997). Synteny relationships were visualized with the software Circos (Krzywinski et al. 2009).

\section{Results}

\section{Marker development and polymorphism analysis}

EST sequences of Petunia were downloaded from GenBank and mined for SSRs. A total of 463 perfect and imperfect SSR motifs were detected. On average, $2.5 \%$ of the ESTs contained at least one microsatellite (one every $23 \mathrm{~kb}$ ). Among the pool of possible SSRs, trinucleotides and mononucleotides were the most abundant (see Supplementary data, Fig. S1). These findings from Petunia are in line with the distribution and frequency of microsatellite motifs in the transcriptomes of maize, rice, and Arabidopsis, as described by Morgante et al. (2002). The over-representation of trinucleotides in the transcriptome is probably explained by the purifying selection acting on repeats whose instability would disrupt the open reading frame. PCR primers were tested on the four Petunia species $P$. axillaris, $P$. exserta, $P$. parodii, and $P$. inflata to check for polymorphisms. Table 1 contains the PCR primers of the microsatellite markers that were polymorphic and that were further genotyped for linkage analysis. CAPS markers were designed on Petunia EST sequences with a putative role in specifying floral traits (e.g., color, scent, nectar, or morphology; Stuurman et al. 2004). AFLP markers were genotyped only on the $\mathrm{BC}_{1}$ cross $P$. axillaris $\times$ $P$. inflata. Eight primer combinations designed with the Pst $\mathrm{I}-$ MseI restriction sites produced 75 polymorphic bands and six primer combinations for the pair EcoRI-MseI amplified 78 polymorphisms. Because most of the AFLP markers clustered around a few loci (data not shown), only 28 nonclustering AFLP bands with high scoring quality were informative and were integrated into the $P$. axillaris $\times P$. inflata map.

The Pt markers developed by relying on tomato positional information greatly improved mapping in Petunia, especially in chromosomes 4 and 7, which historically lacked molecular markers.

\section{Construction of Petunia linkage maps}

Two Petunia genetic maps were obtained by genotyping a set of $173 \mathrm{~F}_{2}$ lines derived from $P$. exserta $\times P$. parodii and $176 \mathrm{BC}_{1}$ lines from $P$. axillaris $\times P$. inflata (Fig. 2, upper panel). Chromosome identity of each linkage group was established by mapping CAPS markers (Table 2) that were used in previous mapping studies (Strommer et al. 2000, 2009). Both maps span the seven chromosomes of the Petunia genome and cover $700 \mathrm{cM}$ in $P$. exserta $\times P$. parodii and $970 \mathrm{cM}$ in $P$. axillaris $\times P$. inflata. The $P$. exserta $\times P$. parodii map consists of 119 markers, with an average marker density of one marker every $6 \mathrm{cM}$, whereas the map of $P$. axillaris $\times P$. inflata was constructed with 125 markers (one every $8 \mathrm{cM}$ ). Using a set of 37 markers common to the two maps, it was possible to compare marker order and recombination rate at 30 orthologous genetic intervals. The two maps retained perfect collinearity of marker order (Fig. 2, lower panel), but substantial differences in recombination frequency at orthologous genetic intervals were observed. Generally, genetic distances in $P$. axillaris $\times P$. inflata were significantly higher than in $P$. exserta $\times P$. parodii (see Supplementary data, Fig. S2). In chromosome 5 the opposite was observed; markers Pm19, ChiA, Gt, and Pm44 cluster in the $P$. axillaris $\times P$. inflata cross, but segregate in the $P$. exserta $\times P$. parodii cross. On average we counted 1.15 crossovers per chromosome in $\mathrm{BC}_{1} P$. axillaris $\times$ $P$. inflata and 1.41 crossovers per chromosome in $\mathrm{F}_{2} P$. exserta $\times P$. parodii (see Supplementary data, Fig. S3). Based on cytological observations, Rees and Durrant (1986) reported on average 1.7 chiasmata per chromosome in Petunia. Assuming transferability of this information, we are covering between $67 \%$ and $82 \%$ of the genome. Up to date, the maps presented here are the most complete, both in terms of the number of markers and genome coverage.

In both crosses, many loci displayed significant segregation distortion for one of the parents. The degree of distortion was stronger in the $P$. exserta $\times P$. parodii population (Fig. 2, lower panel), where the alleles of $P$. exserta were overrepresented for half of the markers. In this cross, distorted markers were mostly localized to chromosomes 1 and 3 and to the top of chromosome 6 . In the $P$. axillaris $\times P$. inflata cross, the distortion was lower and affected a smaller number of 


\begin{tabular}{|c|c|c|c|c|c|}
\hline Chr. & Marker & Forward primer & Reverse primer & Putative function & Polymorphism \\
\hline 1 & PT21 & CAAAGGGGTGGAGCAGCAG & CAACTACCATAAGTTCCTTG & Unknown & Indel \\
\hline 1 & PT55 & GGACTTCCACAGAGAATTGG & GCTGAAACTACATTCAGATAC & Unknown & Indel \\
\hline 1 & PT71 & GTGCTCTCATCCAACATGAG & CCCACGAGTTTGCACCACTAG & Anion channel & Indel \\
\hline 1 & PT93 & ССТCTCTATTCTAAATTGCTTG & GCTGAAACTACATTCAGATAC & Unknown & Indel \\
\hline 1 & РT99 & CTTGATGACTCAGACATATGGC & TCACТTCТСААССАСАТА & BolA-like family & Indel \\
\hline 1 & PT111 & GGACAGTGAGAATCATGCTAC & CTATGGAGGTCAGTCGACCCAC & Armadillo motif & Indel \\
\hline 2 & PT40 & CTCTCTGGTAAGATGGGCTG & TGATGCTCTAATTACTGGC & Unknown & Indel \\
\hline 2 & PT44 & CTTCTCTTGTACTTGGAGG & CACATTTACGCCCAATCTCAG & RAB GDP-dissociation inhibitor & Indel \\
\hline 2 & PT50 & GATGAGCTTGGGGACACCAG & CCAATGCTACACAACACAGTC & Coumarate-CoA ligase & Indel \\
\hline 2 & PT52 & GCAGTGGAAACTAGTGTCAAC & CACCCCACTAATCAACATTAAC & Ethylene forming enzyme & Indel \\
\hline 2 & PT57 & GGAGGCTTTGGTCATGTGAG & TCCTATTCAGATGTCATGA & Peptidase & Indel \\
\hline 2 & PT72 & GTGGTACAAGTACATTGAG & CTCTAGTCTCCTTGCACTAC & Proton-dependent oligopeptide transporter & Indel \\
\hline 2 & PT76 & GTTGCCCATGTTTGGGTG & GCCCTTCACCTTTAAGGG & Unknown & Indel \\
\hline 2 & PT85 & CAATACCTATGGAAGCTCTTAG & GTCCATTGATCTGCCTGAAAC & Exoribonuclease & Indel \\
\hline 2 & РТ96 & CTAACCGGCACAACTAATTGC & ACGTGAAACATCAGCATTG & Unknown & Indel \\
\hline 2 & PT115 & GGGTTGGAGACTCACTCAAC & CGTCAAAAGAACCAATTCT & Aldo/keto reductase & Indel \\
\hline 2 & PT148 & CTTGCCCCCAACAGCTGGTG & GTTAAGAGTTTGAGAGATC & Unknown & Indel \\
\hline 3 & PT39b & СTCCCTTCCGGATCATTGGG & GCAGAATTCATCACACTTCC & Heme binding & Indel \\
\hline 3 & PT102a & GAAGAGCCCTCTAGTATCAG & GCACCGCAGAATGATTTGCTG & Chlorophyll binding & Indel \\
\hline 3 & PT102b & GAAGAGCCCTCTAGTATCAG & GCACCGCAGAATGATTTGCTG & Chlorophyll binding & Indel \\
\hline 3 & PT108 & GCCTAGATCGCATCAGAC & GCTGAAAATTCGAATCATCAGAC & Leucin-rich repeat kinase & Indel \\
\hline 4 & PT27 & CAGGATTGGGATGACGATTGG & CCGTGGTGTATGTACCTCGTG & Peptidoglycan-binding kinase & Indel \\
\hline 4 & PT97 & CTGATGTATGCTAAGCGTGCT & CCAGCAGAATTCATGTCAGC & Cytoskeleton structural constituent & Indel \\
\hline 4 & PT100 & GAACTTGGAGAAGCCGTAAGG & AGATATAACGGCTGTCACC & UDP-glucoronosyl transferase & Indel \\
\hline 4 & PT116 & GGTCACATTCTCAATCTTGG & CATGGAAATAACAAGCTGCTG & Uroporphyrinogen decarboxylase & Indel \\
\hline 5 & PT22 & CAAGCTTCCTGTGCAGTC & TGGAATGACCATTCAACTG & Unknown & Indel \\
\hline 5 & PT26 & CAGATGAGGGATTATCTCC & TTCAGAACTACTTTACA & Carbohydrate kinase & Indel \\
\hline 5 & PT36 & CCTTCAAGCCCCTATGACAAG & CTCATCTCAAACGAAAACC & Calmoduline & Indel \\
\hline 5 & PT37 & CGAGTGGCTATAGCGAGGG & CTCATTCAGCAAAATTCACAAGATC & Unknown & Indel \\
\hline 5 & PT104 & GATGTTGGAAACAGCCTCAAG & GTACATCCGTTTGTCTCTCT & Cinnamyl-alcohol dehydrogenase & Indel \\
\hline 5 & PT113 & GGCACTTCTTCAAGAAATGG & CACATTGAAGTCTTTACAC & Diacylglycerol kinase & Indel \\
\hline 5 & PT114 & GGCGCCTTTCGAAGCACTTTC & CACACCACCGGGTGGCTCGCC & Unknown & Indel \\
\hline 5 & PT140 & GGAGCTGAGAAATCAGTGT & CTCCACTAAAGTGGGAAGGAG & ATP synthase & Indel \\
\hline 6 & PT25 & CAGAGCCTTGGGAGCTAGAGC & AGAAGCTTGTGGCAGCC & Unknown & Indel \\
\hline 6 & PT84 & CAAGGAGAGCTTATGTGAGC & TGTGAAGATACAGCTACCAG & Unknown & Indel \\
\hline 6 & PT105 & GCATCTTGTTTAGGACAACC & CAAGTAATGAATCGCTAAGTTCC & Unknown & Indel \\
\hline 6 & PT110 & GGAATTGCAGAGTGGCAGAGC & CAAAAGCCAAAACTCATACG & Nucleoside diphosphate kinase & Indel \\
\hline 6 & PT149 & GAGATGTACTAGTACTATG & GTACATTGTTGTCGAAC & Unknown & Indel \\
\hline 7 & PT3 & СТTCCССТCСTTCAACGCATGTACG & CGGAAACGGCCTCTCACCC & Unknown & Indel \\
\hline 7 & PT5 & CAGGATCCTAAGTATTGGAC & GCATGACTCCTTTATCGAC & Exonuclease & DpnII \\
\hline 7 & PT6 & CTCGGTCTGGACTTGATTCAG & CCTTTGTAAGATAATCCCCTG & Unknown & Indel \\
\hline 7 & PT7 & GTGGAGTCTGCATCTATGG & CTTCAGATCATCCTCAGTGAG & Unknown & NsiI \\
\hline 7 & PT8 & CCTTAGGACCTGCATCACCC & CAGCGGCTATCTTTGGAGC & Heat-shock protein & Indel \\
\hline 7 & PT11 & GAATGTGGATGTGGACCTCG & GCTGCTCCCCTCGTCAGATCC & Superoxide dismutase & $R s a \mathrm{I}$ \\
\hline 7 & PT13 & CATGGCCTTGATGTCTCAGG & CCGCGAAGAAGTATGCAC & Glutamate-cysteine ligase & Indel \\
\hline 7 & PT15 & CTAAAGATTCCCATGAATCAGC & GGGGAAGATGTAGTTTTATAACC & Unknown & Indel \\
\hline 7 & PT30 & CCAAGTGATTCCACCATCTC & GAGATATTCCACCACCC & RNA polymerase & Indel \\
\hline 7 & PT39a & CTCCCTTCCGGATCATTGGG & GCAGAATTCATCACACTTCC & Heme binding & Indel \\
\hline 7 & PT87 & CATGTGATTCTATAATCCGAG & TGAGGCAATCCCGGGTCTTTTG & Gamma carbonic anydrase & Indel \\
\hline 7 & PT134 & CCAAGTTACTAGGAGTACC & GTAATGCCCAATGGTTC & $O$-Methyltransferase & Indel \\
\hline
\end{tabular}

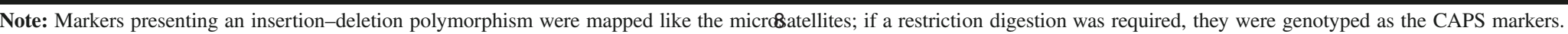


Fig. 2. Genetic maps of Petunia interspecific crosses. Linkage maps of Petunia obtained from two interspecific crosses. For each chromosome, the Petunia axillaris $\times$ Petunia inflata map is represented on the left (denoted by axin) and the Petunia exserta $\times$ Petunia parodii on the right (denoted by expa). The markers in bold are shared between the two maps. The diagram below each chromosome displays the segregation distortion at each marker locus. Distortion towards $P$. axillaris or $P$. exserta is to the left and towards $P$. inflata or $P$. parodii is to the right. Black horizontal lines correspond to markers that are not significantly distorted. Red color refers to a distortion in favour of $P$. exserta or $P$. inflata. The length of the line is proportional to the degree of the distortion, as indicated in the legend. Dotted lines in the middle connect the markers shared between the two crosses.
$\mathrm{cM}$
Ch.1 axin
Ch.1 expa
Ch.2 axin
Ch.2 expa
Ch.3 axin
Ch.3 expa
Ch.4 axin
Ch.4 expa
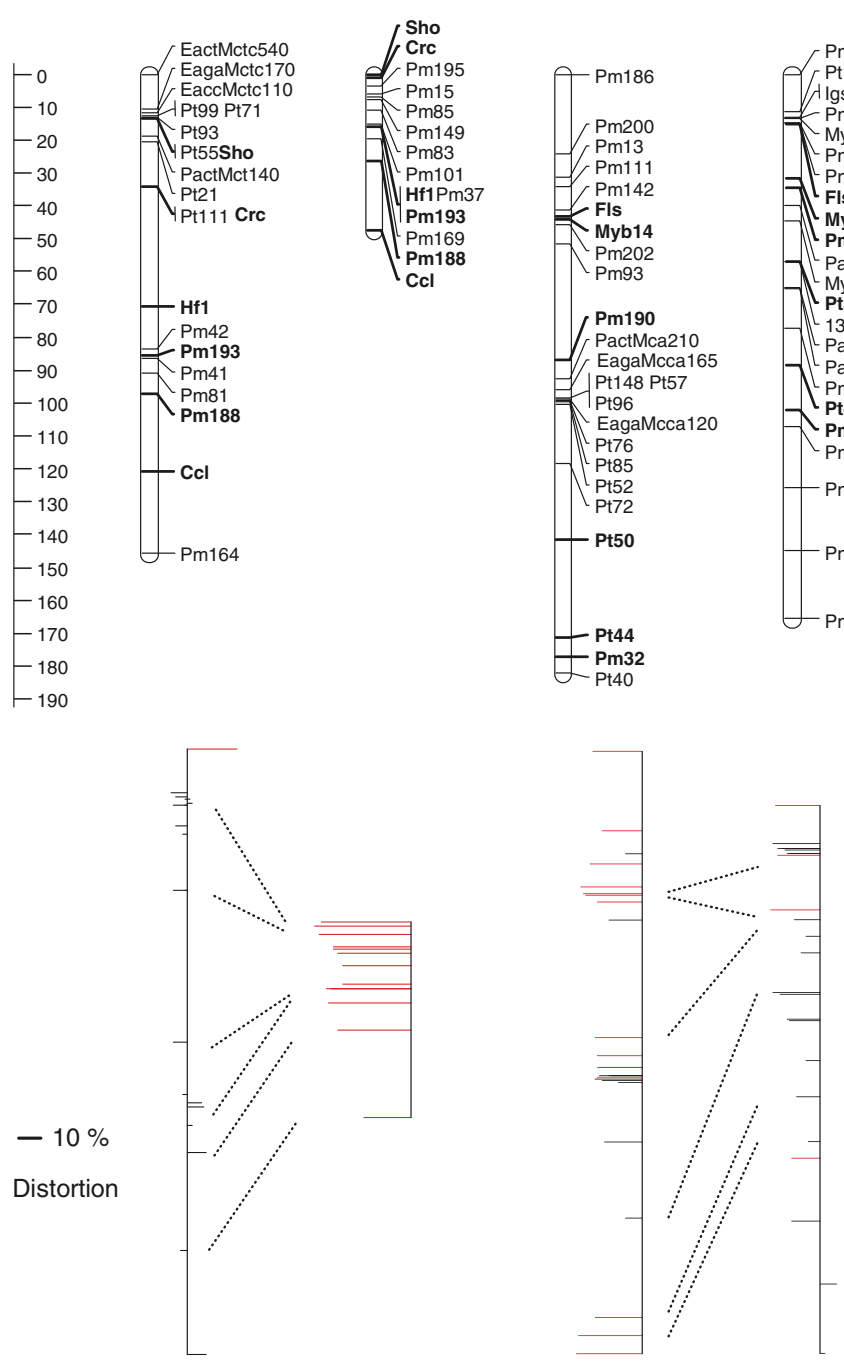

markers $(30 \%)$, mostly in chromosome 2 towards the $P$. axillaris alleles and in chromosome 3 towards the $P$. inflata alleles. Interestingly, the chromosomal regions subject to segregation distortion were different in the two crosses.

\section{Comparative mapping of Petunia and tomato reveals a complex mosaic of rearrangements}

We tested synteny between the two genetic maps of Petunia and the physical map of tomato (assembly 2.10, solgenomics.net, Müller et al. 2005) with a BLASTN search of the Petunia marker sequences in the tomato genome database. Conservation of macrosynteny within the Solanaceae has been described between the genomes of tomato, potato, pepper, eggplant, and diploid tobacco (reviewed by $\mathrm{Wu}$ and Tanksley 2010). The genomes of these solanaceous crops all have the same chromosome number $(2 n=24)$. In contrast,
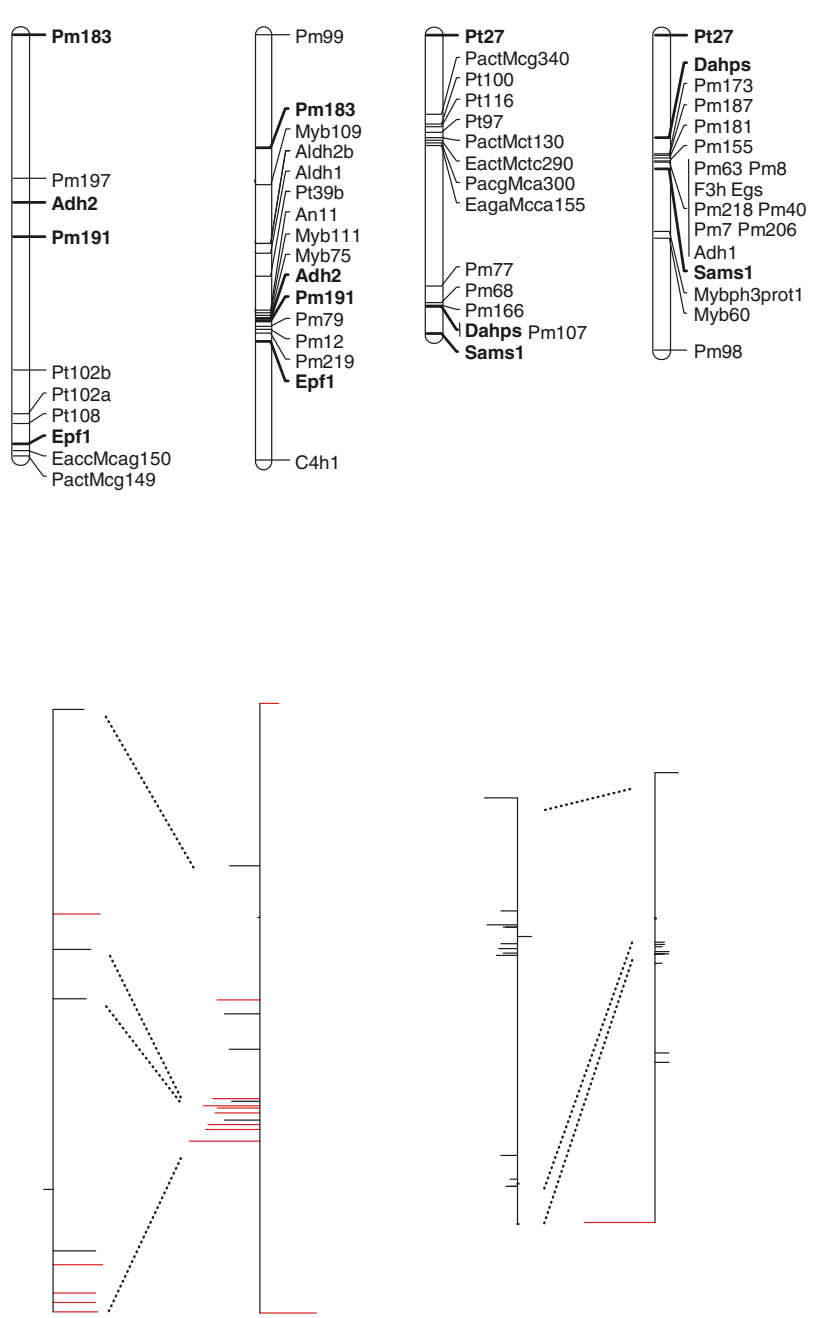

Petunia has a chromosome number of $2 n=14$, necessitating a number of rearrangements. A tabular summary of the BLASTN output is provided in the Supplementary data (Table S1). The pattern of synteny varies greatly for different chromosomes (Fig. 3). Chromosome 7 of Petunia retains most of the markers from the tomato chromosome 8. Chromosome 5 retains most of the markers from the tomato chromosome 12. Chromosome 1 and 6 of Petunia contain segments of the tomato chromosomes 5 and 6 , and 1 and 9, respectively. Chromosomes 3 and 4 of Petunia retain synteny with chromosomes 3 and 4 of tomato, but only for a segment of the terminal portions of the long arms. Within orthologous syntenic blocks, we observed little correlation between genetic distances of Petunia and physical distances in tomato. In Petunia chromosome 2 synteny was more disrupted. This chromosome contains segments of tomato chromosomes 2, 7 , 
Fig. 2. (concluded).
$\mathbf{c M}$
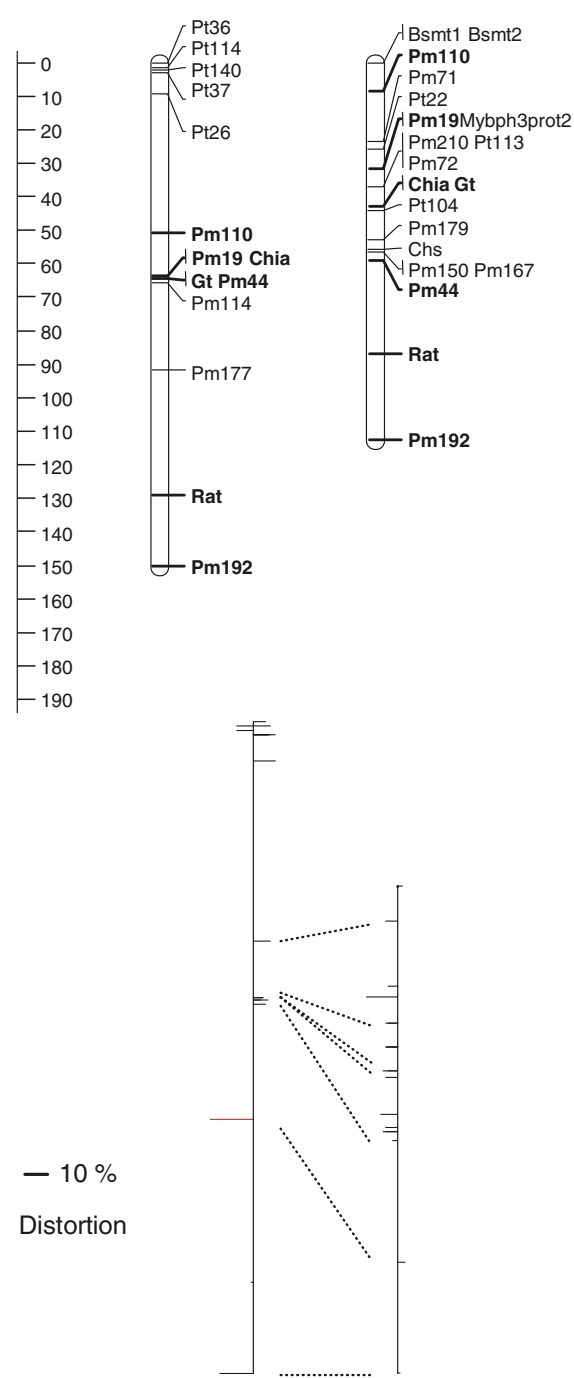

8, and 10. Most of these observations are consistent in the two crosses; small-scale incongruence (e.g., in chromosome 4) is explained by the presence of different marker sequences in the two maps.

\section{Discussion}

\section{Distortion of the segregation frequencies as a result of recent species radiation}

For half of the marker loci mapped in the P. exserta $\times$ $P$. parodii cross we observed significantly more alleles of $P$. exserta. For all loci but one (Pm99), distortion was towards $P$. exserta alleles. In chromosomes 1 and 6, 40\% more $P$. exserta alleles were observed than expected. Conversely, segregation distortion in the $P$. axillaris $\times P$. inflata cross was more localized, more moderate, and in the direction of both parents. Distortion is localized to specific chromosomal segments that are different in the two crosses. This indicates that distortion originates from species-specific locus interactions. These interactions may be prezygotic, resulting from differences in the growth rates of recombinant pollen
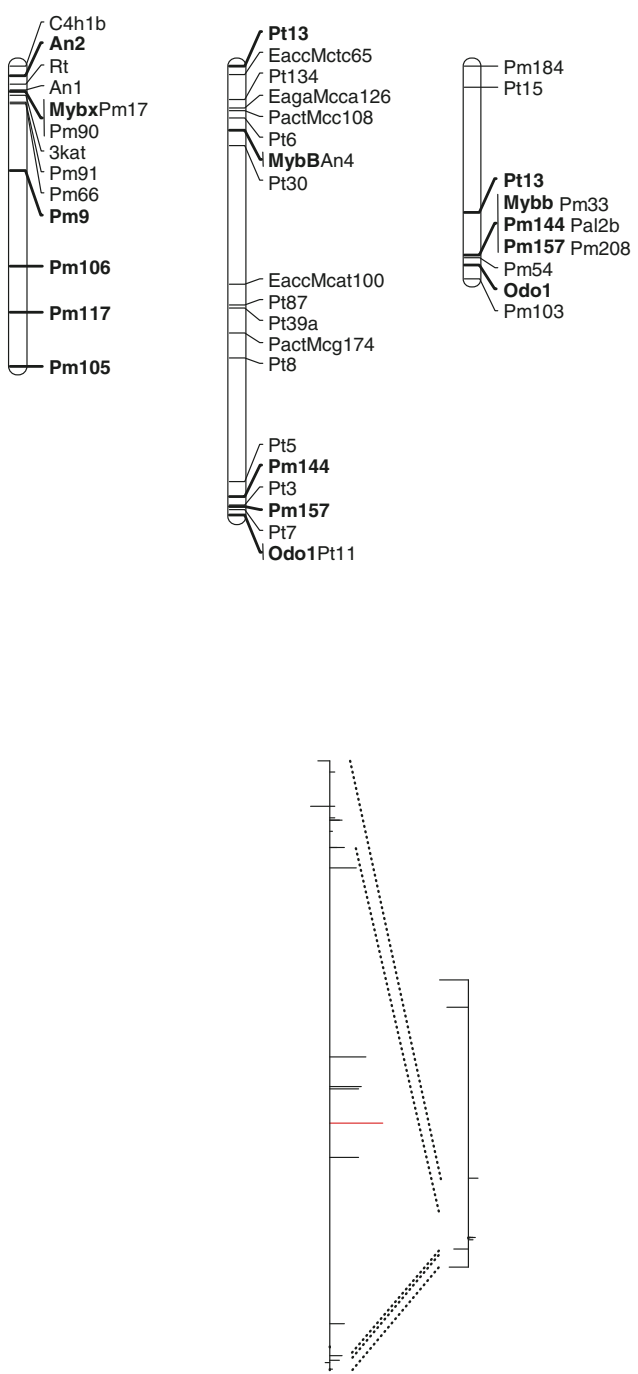

in the styles of the $F_{1}$ cross. Because the $F_{1}$ pollen has a recombinant genotypic constitution, the loci influencing pollen growth would be segregating. Thus, distortion affects only specific chromosomal segments and not all of the genome. Distortion may also result from postzygotic selection against specific allelic combinations that determine lower fitness in interspecific hybrids. While growing the recombinant plants, several seeds failed to germinate and the seedlings differed greatly in growth rate, fitness, and survival. Segregation distortion in interspecific hybrids has previously been observed in Petunia (Robbins et al. 1995; Strommer et al. 2000) and more generally within the Solanaceae and other plant families (Zamir and Tadmor 1986). In Arabidopsis, temperature-dependent lethal allelic combinations of the Bateson-Dobzhansky-Müller type were ascribed to autoimmune-like responses (Bomblies et al. 2007). Petunia exserta is a rare species endemic to a restricted area $\left(500 \mathrm{~km}^{2}\right)$ of Brazil. In this area it grows sympatrically with the highly abundant $P$. axillaris and interspecific hybridization has been observed (Lorenz-Lemke et al. 2006). From an evolutionary perspective, hybridization represents a threat to spe- 
Fig. 3. Synteny relationship with the tomato genome. The seven chromosomes of Petunia are depicted with different colors. Lines of the same color of the chromosomes connect Petunia marker sequences with their physical position in the genome of tomato. The chromosomes of tomato are represented in grey and have been reordered to minimize line overlapping. The synteny relationships with tomato have been plotted independently for the maps of Petunia axillaris $\times$ Petunia inflata $(a)$ and Petunia exserta $\times$ Petunia parodii $(b)$. The units on the chromosomes are given in centimorgans for Petunia and megabases for tomato.

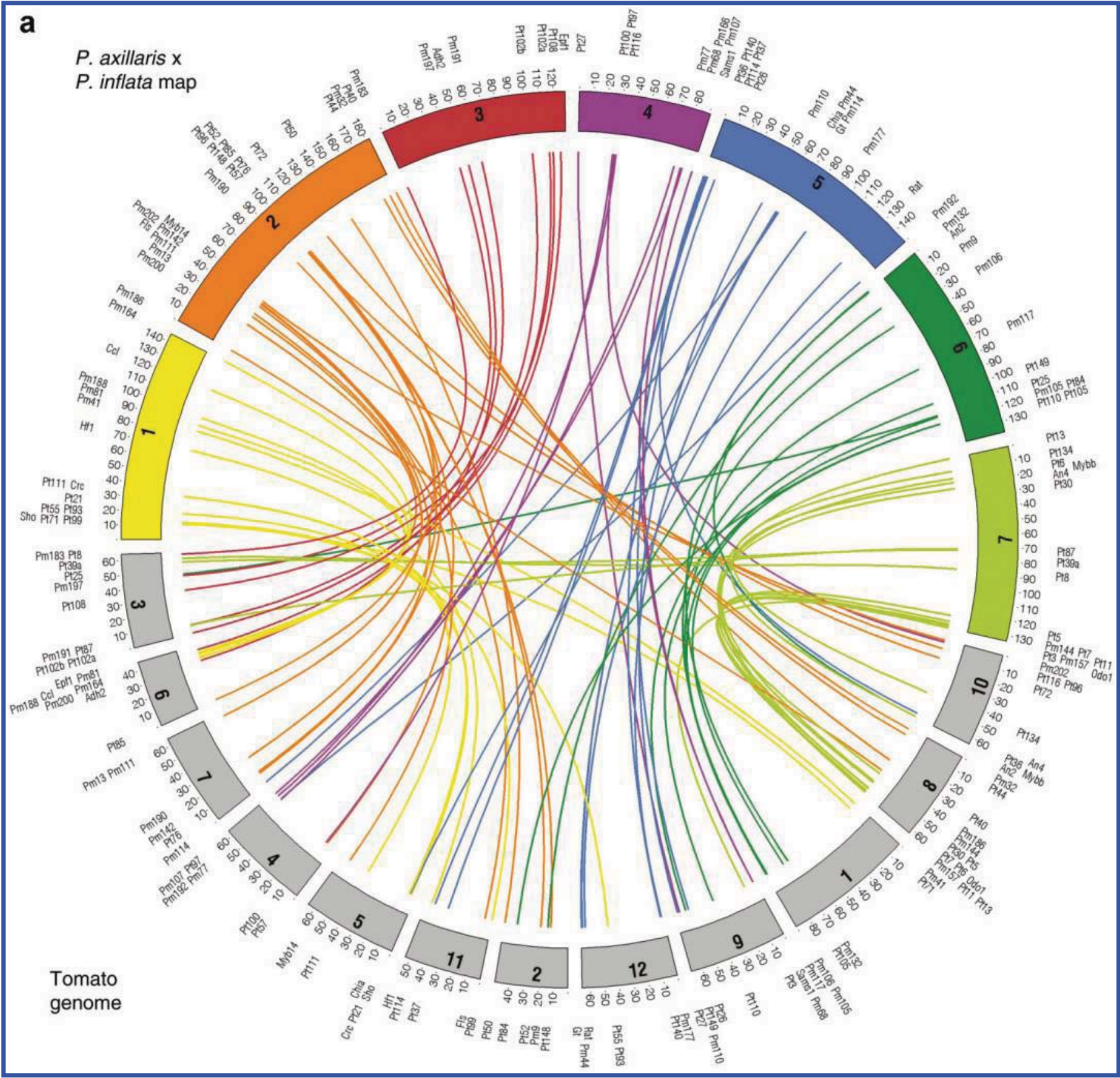

cies identity and segregation distortion in favour of $P$. exserta may be an advantage contributing to the maintenance of species identity.

\section{Genetic recombination in Petunia is influenced by the genetic background}

In the two interspecific crosses we observed significant differences in recombination frequency. Previous genetic maps of Petunia were done with accessions of the garden line P. hybrida (Strommer et al. 2000, 2002; Stuurman et al.
2004). In these previous studies great differences in recombination frequency at orthologous genetic intervals were also observed. While counting recombination frequency in different crosses, Cornu et al. (1989) postulated the presence of a major modulator of recombination $(R m l)$ present on chromosome 2 of the $P$. hybrida line St43. This locus is described as dominant with complete penetrance. It is not clear whether this gene of $P$. hybrida can be traced back to the $P$. axillaris or $P$. inflata germplasm. However, as $P$. parodii has a genetic background very similar to that of $P$. axillaris, but exhibits 
Fig. 3. (concluded).

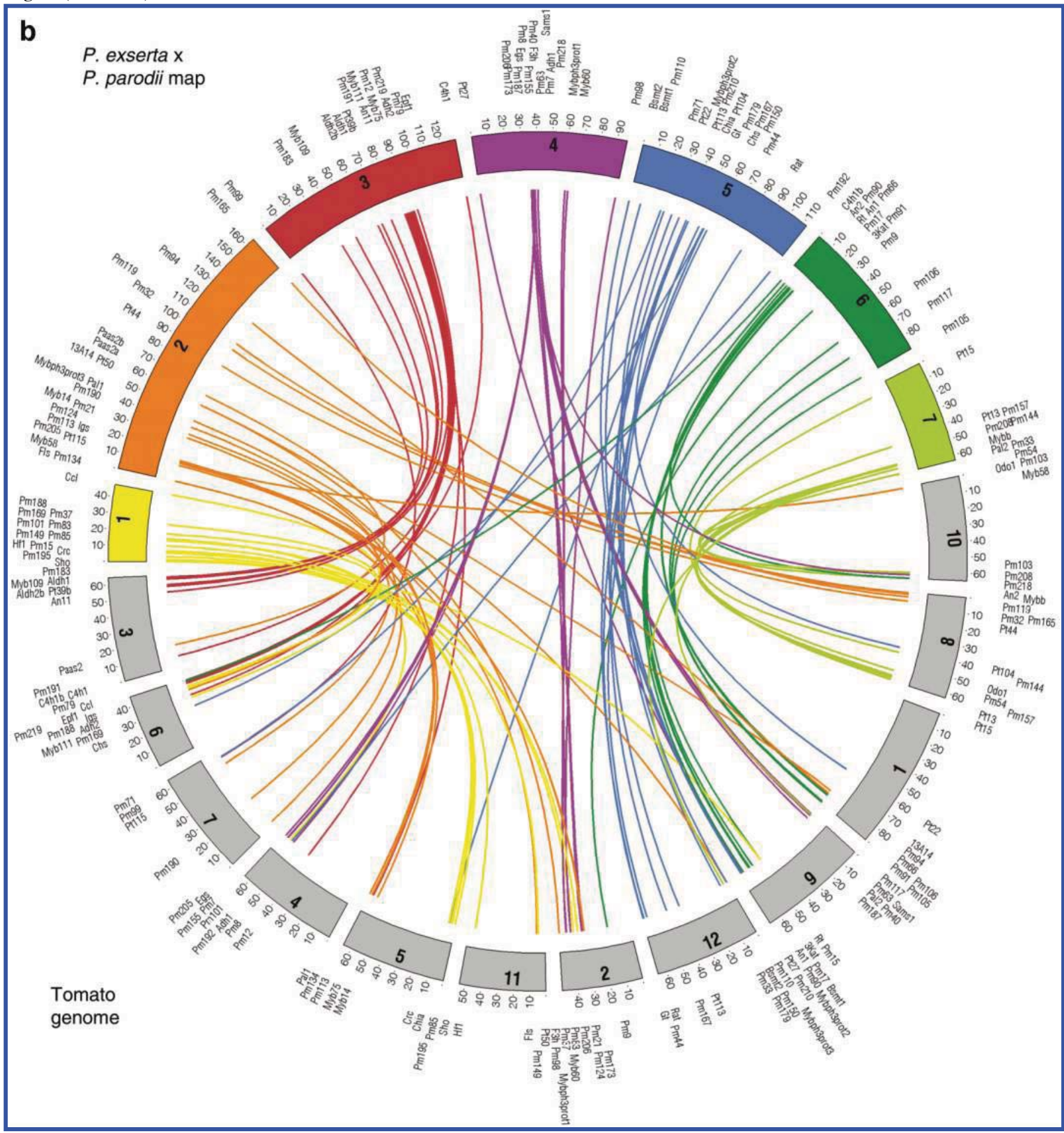

large differences in recombination rate, the $R m 1$ locus is more likely to have been derived from $P$. inflata; this would explain the higher recombination rate observed in the $P$. axillaris $\times P$. inflata cross. Robbins et al. (1995), using T-DNA insertions, observed that extensive suppression of recombination occurred in hybrid genetic backgrounds, but recombination levels were three times higher in an inbred background. Recombination suppression was therefore attributed to physical rearrangements in hybrids. Physical evidence for suppres- sion of recombination was later confirmed by ten Hoopen et al. (1996) with fluorescence in situ hybridization. To some extent we also observed that in orthologous genetic intervals recombination can vary greatly. In some cases suppression of recombination led to complete clustering of genetic markers. In chromosomes 2,5 , and 7 of the $P$. axillaris $\times P$. inflata population, clusters of genetic markers occurred in more than one chromosomal segment, indicating that clustering cannot be explained only by the presence of the centromere. 
An intriguing hypothesis is the presence of residual paleocentromeres or heterochromatic regions remaining after chromosome fusion during the radiation of the Petunioideae clade. We speculate that $x=12$ may represent the ancestral chromosome number of the Solanaceae family. Some of these chromosome fusions in Petunia may have occurred only recently, as the Petunia sister taxon Calibrachoa has the karyotype $n=x=9$ (reviewed by Stehmann et al. 2009). Generally, chromosome fragmentation and fusion are known to happen and represent an important speciation factor in plants. However, more cytological work is needed to shed light on the role of chromosomal rearrangements for species radiation within the Solanaceae.

Despite the differences in recombination rate observed between the two crosses, the order of the 37 shared markers was identical, indicating that the genome structure within the genus is well conserved. These results are consistent with the findings from Strommer et al. (2000, 2002), where despite big differences in recombination frequency, marker order between different crosses was largely retained.

\section{Distribution of Petunia genic microsatellites and their conservation in tomato}

In the Petunia transcriptome, we detected on average one microsatellite every $23 \mathrm{~kb}$. Generally, microsatellites in plants are preferentially associated with nonrepetitive DNA and the number of microsatellite motifs detected in expressed sequences is considerably higher than that observed in intergenic DNA (Morgante et al. 2002). Within the Arabidopsis transcriptome, the untranslated regions (UTRs) are the sequences with the highest dinucleotides abundance, whereas trinucleotides are most abundant in coding sequences (Morgante et al. 2002). Using unigenes and predicted proteins from tomato, we could annotate the position of 29 SSRs in the Petunia transcripts (see Supplementary data, Fig. S4). Although the data set is small for a conclusive statement, the distribution observed in Petunia is similar to what Morgante et al. (2002) found in Arabidopsis. Despite 35 million years of divergent evolution with tomato (Wang et al. 2008), 60\% of the Petunia microsatellites were also detected in the orthologous tomato EST, either as fully developed motifs or as short protorepeats. Microsatellite positions were better conserved in the coding portion of the ESTs. EST-microsatellites may play a role in the generation of allelic variation, e.g., by modifying the protein primary structure or by altering mRNA stability. In animals, numerous studies correlate the generation of rapid phenotypic variation with the instability of EST-microsatellites (Sutherland and Richards 1995; Fondon and Garner 2004; Hammock and Young 2005). Although at present a thorough investigation does not exist, microsatellites are likely to provide a substrate for rapid phenotypic variation also in plants.

\section{Conclusions}

We have constructed two linkage maps of Petunia with gene-derived markers using two interspecific crosses, and we report for the first time the development and mapping of multiallelic microsatellite markers of Petunia. Genetic analysis has shown that the structure of the Petunia genome is well conserved within the genus. Local segregation distortion within the interspecific crosses hints at partial genetic barriers that arose after recent speciation. Comparative mapping with tomato suggests that numerous genomic rearrangements occurred during the radiation of these two Solanaceae species. Given the decay of synteny between tomato and Petunia, the usefulness of the tomato genome as a template for comparative genomics is limited to a few chromosomal segments, and a Petunia genome sequence would be needed to support genomic research on this model plant. A Petunia sequencing initiative has been undertaken. The maps reported here will facilitate the assembly of its large genome.

\section{Acknowledgments}

We thank E. Jakab and M. Grémillon for their technical assistance, and C. Ball and N. Signer for greenhouse help. We gratefully acknowledge the contribution of the Solanaceae Genomics Network for maintaining an online database with the tomato genome sequences. Finally, we thank R. Koes and F. Quattrocchio for generously supplying materials and T. Gerats for a critical reading of the manuscript. This work was supported by the University of Bern and by the National Centre for Competence in Research "Plant Survival".

\section{References}

Abajian, C. 1994. SPUTNIK [online]. Available from cbib.ubordeaux2.fr/pise/sputnik.html [accessed 19 October 2008].

Altschul, S.F., Madden, T.L., Schäffer, A.A., Zhang, J.H., Zhang, Z., Miller, W., and Lipman, D.J. 1997. Gapped BLAST and PSIBLAST: a new generation of protein database search programs. Nucleic Acids Res. 25(17): 3389-3402. doi:10.1093/nar/25.17. 3389. PMID:9254694.

Bentolila, S., Alfonso, A.A., and Hanson, M.R. 2002. A pentatricopeptide repeat-containing gene restores fertility to cytoplasmic male-sterile plants. Proc. Natl. Acad. Sci. U.S.A. 99(16): 1088710892. doi:10.1073/pnas.102301599. PMID:12136123.

Bomblies, K., Lempe, J., Epple, P., Warthmann, N., Lanz, C., Dangl, J.L., and Weigel, D. 2007. Autoimmune response as a mechanism for a Dobzhansky-Müller-type incompatibility syndrome in plants. PLoS Biol. 5(9): e236. doi:10.1371/journal.pbio.0050236.

Broman, K.W., and Sen, S. 2009. A guide to QTL mapping with R/ qtl. Springer, New York.

Conner, A.J., Albert, N.W., and Deroles, S.C. 2009. Transformation and regeneration of Petunia. In Petunia: evolutionary, developmental and physiological genetics. 2nd ed. Edited by T. Gerats and J. Strommer. Springer, New York. pp. 395-416.

Cornu, A., Farcy, E., and Mousset, C. 1989. A genetic basis for variations in meiotic recombination in Petunia hybrida. Genome, 32: 46-53. doi:10.1139/g89-409.

Fondon, J.W., III, and Garner, H.R. 2004. Molecular origins of rapid and continuous morphological evolution. Proc. Natl. Acad. Sci. U. S.A. 101(52): 18058-18063. doi:10.1073/pnas.0408118101. PMID:15596718.

Gerats, T., and Strommer, J. 2009. Petunia: evolutionary, developmental and physiological genetics. 2nd ed. Springer, New York.

Gerats, A.G.M., Huits, H., Vrijlandt, E., Maraña, C., Souer, E., and Beld, M. 1990. Molecular characterization of a nonautonomous transposable element (dTphl) of Petunia. Plant Cell, 2(11): 11211128. doi:10.1105/tpc.2.11.1121. PMID:1967052.

Gerats, A.G.M., Souer, E., Kroon, J., McLean, M., Farcy, E., and Maizonnier, D. 1993. Petunia hybrida. In Genetic maps: locus maps of complex genomes. 6th ed. Edited by S. O'Brien. Cold Spring Harbor Laboratory Press, New York. pp. 6.13-6.23. 
Hammock, E.A.D., and Young, L.J. 2005. Microsatellite instability generates diversity in brain and sociobehavioral traits. Science, 308(5728): 1630-1634. doi:10.1126/science.1111427. PMID: 15947188 .

Kosambi, D.D. 1944. The estimation of map distances from recombination values. Ann. Eugen. 12: 172-175.

Krzywinski, M., Schein, J.E., Birol, I., Connors, J., Gascoyne, R., Horsman, D., 2009. Circos: an information aesthetic for comparative genomics. Genome Res. 19(9): 1639-1645. doi:10. 1101/gr.092759.109. PMID:19541911.

Lander, E.S., Green, P., Abrahamson, J., Barlow, A., Daly, M.J., Lincoln, S.E., and Newberg, L.A. 1987. MAPMAKER: an interactive computer package for constructing primary genetic linkage maps of experimental and natural populations. Genomics, 1(2): 174-181. doi:10.1016/0888-7543(87)90010-3. PMID: 3692487.

Lorenz-Lemke, A.P., Mäder, G., Muschner, V.C., Stehmann, J.R., Bonatto, S.L., Salzano, F.M., and Freitas, L.B. 2006. Diversity and natural hybridization in a highly endemic species of Petunia (Solanaceae): a molecular and ecological analysis. Mol. Ecol. 15 (14): 4487-4497. doi:10.1111/j.1365-294X.2006.03100.x. PMID: 17107478 .

McCubbin, A.G., Zuniga, C., and Kao, T. 2000. Construction of a binary bacterial artificial chromosome library of Petunia inflata and the isolation of large genomic fragments linked to the selfincompatibility ( $S$-) locus. Genome, 43(5): 820-826. doi:10.1139/ gen-43-5-820. PMID:11081972.

Mishiba, K.I., Ando, T., Mii, M., Watanabe, H., Kokubun, H., Hashimoto, G., and Marchesi, E. 2000. Nuclear DNA content as an index character discriminating taxa in the genus Petunia sensu Jussieu (Solanaceae). Ann. Bot. 85(5): 665-673. doi:10.1006/ anbo.2000.1122.

Morgante, M., Hanafey, M., and Powell, W. 2002. Microsatellites are preferentially associated with nonrepetitive DNA in plant genomes. Nat. Genet. 30(2): 194-200. doi:10.1038/ng822. PMID:11799393.

Müeller, L.A., Solow, T.H., Taylor, N., Skwarecki, B., Buels, R., Binns, J., 2005. The SOL Genomics Network: a comparative resource for Solanaceae biology and beyond. Plant Physiol. 138 (3): 1310-1317. doi:10.1104/pp.105.060707. PMID:16010005.

Murray, M.G., and Thompson, W.F. 1980. Rapid isolation of high molecular weight plant DNA. Nucleic Acids Res. 8(19): 43214326. doi:10.1093/nar/8.19.4321. PMID:7433111.

Puerta, A.R., Ushijima, K., Koba, T., and Sassa, H. 2009 Identification and functional analysis of pistil self-incompatibility factor HT-B of Petunia. J. Exp. Bot. 60(4): 1309-1318. doi:10. 1093/jxb/erp005. PMID:19282427.

R Development Core Team. 2010. R: A language and environment for statistical computing. R Foundation for Statistical Computing, Vienna, Austria.

Rees, H., and Durrant, A. 1986. Recombination and genome size. Theor. Appl. Genet. 73(1): 72-76. doi:10.1007/BF00273721.

Reid, M., Chen, J.C., and Jiang, C.-Z. 2009. Virus-induced gene silencing for functional characterization of genes in Petunia. In Petunia: evolutionary, developmental and physiological genetics. 2nd ed. Edited by T. Gerats and J. Strommer. Springer, New York. pp. 395-416.

Robbins, T.P., Gerats, A.G.M., Fiske, H., and Jorgensen, R.A. 1995.
Suppression of recombination in wide hybrids of Petunia hybrida as revealed by genetic mapping of marker transgenes. Theor. Appl. Genet. 90(7-8): 957-968. doi:10.1007/BF00222909.

Schuelke, M. 2000. An economic method for the fluorescent labeling of PCR fragments. Nat. Biotechnol. 18(2): 233-234. doi:10.1038/ 72708. PMID:10657137.

Stehmann, J.R., Lorenz-Lemke, A.P., Freitas, L.B., and Semir, J. 2009. The genus Petunia. In Petunia: evolutionary, developmental and physiological genetics. 2nd ed. Edited by T. Gerats and J. Strommer. Springer, New York. pp. 1-28.

Strommer, J., Gerats, A.G.M., Sanago, M., and Molnar, S.J. 2000. A gene-based RFLP map of Petunia. Theor. Appl. Genet. 100(6): 899-905. doi:10.1007/s001220051368.

Strommer, J., Peters, J., Zethof, J., De Keukeleire, P., and Gerats, T. 2002. AFLP maps of Petunia hybrida: building maps when markers cluster. Theor. Appl. Genet. 105(6-7): 1000-1009. doi:10.1007/s00122-002-1009-y. PMID:12582927.

Strommer, J., Peters, J.L., and Gerats, T. 2009. Genetic recombination and mapping in Petunia. In Petunia: evolutionary, developmental and physiological genetics. 2nd ed. Edited by T. Gerats and J. Strommer. Springer, New York. pp. 395-416.

Stuurman, J., and Kuhlemeier, C. 2005. Stable two-element control of dTphl transposition in mutator strains of Petunia by an inactive ACT1 introgression from a wild species. Plant J. 41(6): 945-955. doi:10.1111/j.1365-313X.2005.02340.x. PMID:15743456.

Stuurman, J., Hoballah, M.E., Broger, L., Moore, J., Basten, C., and Kuhlemeier, C. 2004. Dissection of floral pollination syndromes in Petunia. Genetics, 168(3): 1585-1599. doi:10.1534/genetics.104. 031138. PMID:15579709.

Sutherland, G.R., and Richards, R.I. 1995. Simple tandem DNA repeats and human genetic disease. Proc. Natl. Acad. Sci. U.S.A. 92(9): 3636-3641. doi:10.1073/pnas.92.9.3636. PMID:7731957.

ten Hoopen, R., Robbins, T.P., Fransz, P.F., Montijn, B.M., Oud, O., Gerats, A.G.M., and Nanninga, N. 1996. Localization of T-DNA insertions in Petunia by fluorescence in situ hybridization: physical evidence for suppression of recombination. Plant Cell, 8 (5): 823-830. doi:10.1105/tpc.8.5.823. PMID:12239403.

Vandenbussche, M., Janssen, A., Zethof, J., van Orsouw, N., Peters, J., van Eijk, M.J.T., 2008. Generation of a 3D indexed Petunia insertion database for reverse genetics. Plant J. 54(6): 1105-1114. doi:10.1111/j.1365-313X.2008.03482.x. PMID:18346192.

Voorrips, R.E. 2002. MapChart: software for the graphical presentation of linkage maps and QTLs. J. Hered. 93(1): 77-78. doi:10. 1093/jhered/93.1.77. PMID:12011185.

Vos, P., Hogers, R., Bleeker, M., Reijans, M., van de Lee, T., Hornes, M., Friters, A., Pot, J., Paleman, J., Kuiper, M., and Zabeau, M. 1995. AFLP: a new technique for DNA fingerprinting. Nucleic Acids Res. 23(21): 4407-4414. doi:10.1093/nar/23.21.4407. PMID:7501463.

Wang, Y., Diehl, A., Wu, F.N., Vrebalov, J., Giovannoni, J., Siepel, A., and Tanksley, S.D. 2008. Sequencing and comparative analysis of a conserved syntenic segment in the Solanaceae. Genetics, 180 (1): 391-408. doi:10.1534/genetics.108.087981. PMID:18723883.

Wu, F.N., and Tanksley, S.D. 2010. Chromosomal evolution in the plant family Solanaceae. BMC Genomics, 11(1): 182. doi:10. 1186/1471-2164-11-182. PMID:20236516.

Zamir, D., and Tadmor, Y. 1986. Unequal segregation of nuclear genes in plants. Bot. Gaz. 147(3): 355-358. doi:10.1086/337602. 